\title{
Empirical Compliance Equations for Constant Rectangular Cross Section Flexure Hinges and Their Applications
}

\author{
Tiemin Li, Yunsong Du, Yao Jiang, and Jinglei Zhang \\ Manufacturing Engineering Institute, Department of Mechanical Engineering, Tsinghua University, Beijing 100084, China \\ Correspondence should be addressed to Yunsong Du; duyunsongwei@163.com
}

Received 7 November 2015; Accepted 13 March 2016

Academic Editor: Dane Quinn

Copyright (C) 2016 Tiemin Li et al. This is an open access article distributed under the Creative Commons Attribution License, which permits unrestricted use, distribution, and reproduction in any medium, provided the original work is properly cited.

\begin{abstract}
This paper presents the derivation of empirical compliance equations of the constant rectangular cross section flexure hinge. The stress concentration caused by changes in cross section is analyzed based on finite element analysis results for the purpose of overcoming compliance calculation errors. It shows that the stress concentration has great influence on axial compliance calculation, while it has little influence on shear and bending compliance calculation. Then empirical compliance equations with a relative wide range of $h / L$ and $t / L$ are derived based on the exponential model in conjunction with consideration of all geometrical parameters of flexure hinges and the influence of the stress concentration on axial compliance calculation. Finally, in order to verify the validity of the empirical equations, the input/output compliance and displacement amplification ratios of bridge-type microdisplacement amplification mechanisms are analyzed. Meanwhile, an experimental platform of displacement amplification mechanisms is set up. The experimental results and finite element method (FEM) values are in good agreement with the theoretical arithmetic, which demonstrates the accuracy of the empirical compliance equations. It provides a reference point for further studies on the design and optimization of flexure hinges and compliant mechanisms.
\end{abstract}

\section{Introduction}

A flexure hinge is actually a complex spring element that can respond to and transmit both rotation and translation. Compared with the classical joints, flexure hinges have many advantages such as no clearance, no friction losses, no need for lubrication, compactness, and ease of fabrication [1, 2]. Flexure hinges have been widely used in applications such as gyroscopes, accelerometers, balance scales, missilecontrol nozzles, multiplying linkages, microgrippers [3-5], and micro-/nanopositioning stages [6-8]. Micromanufacturing, microsystem assembly, biological cell manipulation in biotechnology, and MEMS (microelectromechanical system) increase the demand of flexure hinges which are used to manipulate microscale objects and perform very small motions [9].

Compliance is an essential parameter that represents the relationship between loads and deflections. A compliant mechanism normally uses a few flexure hinges to provide the desired fine motions in various directions. Obviously, the accuracy of a compliant mechanism relies on the precision of a flexure hinge model. Therefore, compliance (the inverse of stiffness) equations of flexure hinges are demanded to be as accurate as possible to reduce the accumulated modeling errors of hinges, subsequently reducing the overall modeling error of compliant mechanisms [10, 11].

There have been many methods adopted to derive the compliance equations of flexure hinges [12-14], including the integration of linear differential equations of a beam, Castigliano's second theorem, inverse conformal mapping, and empirical equations formed from FEM. It can be dated back to 1965; Paros [12] firstly presented the compliance-based approach to the symmetric circular and right circular flexure hinges by giving the compliance equations. Particularly, the angles and linear deflections produced on all three axes were expressed in terms of the corresponding external loading. Lobontiu et al. $[15,16]$ derived the closed-form equations of the compliance for corner-filleted flexure hinges using Castigliano's second theorem. Similar to elliptical flexure hinges, corner-filleted flexure hinges also ranged within the domain 


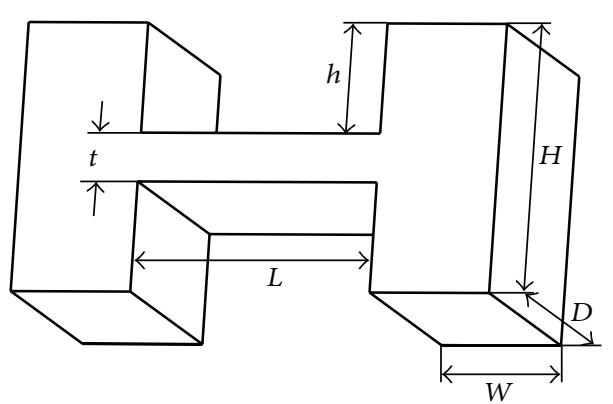

(a)

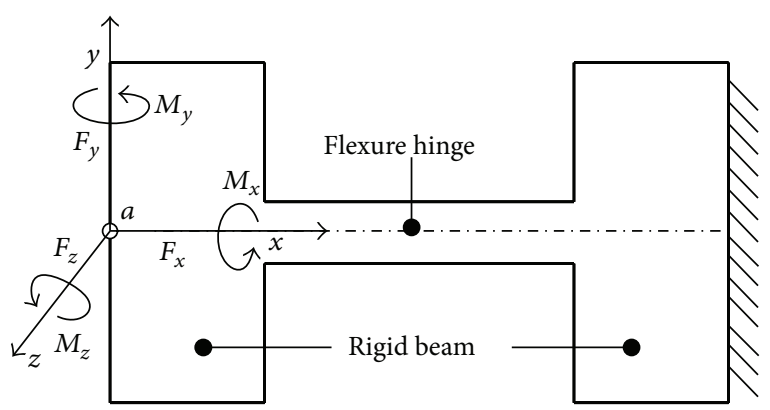

(b)

FIGURE 1: The constant rectangular cross section flexure hinge: (a) dimensions; (b) loads.

confined by right circular flexure hinges and leaf flexure hinges in terms of compliance. It was noted that cornerfilleted flexure hinges were more compliant and induced lower stresses but were less precise in rotation compared with right circular flexure hinges. Tseytlin [17] presented the closed-form equations of the compliance for monolithic flexure hinges with circular, elliptical sections by using the inverse conformal mapping method, and the predictions of the developed models were likely to be much closer to the FEM analysis and the experimental data. Acer and Sabanovic [18] compared the analytical in-plane compliance calculation methods for single-axis circular flexure hinges with FEM, and these comparisons were also made for varying geometrical parameters. While designing a compliant mechanism, the comparisons could give us advices on selecting the right compliance calculation method which was less time-consuming and help us choose the right geometrical parameters by showing their effects on the compliance. Meng et al. [19] derived three stiffness/compliance equations for corner-filleted flexure hinges, which were formulated based on FEM analysis results for the purpose of overcoming the investigated limitations. The empirical equations could enlarge the range of rate of thickness to length and ensure the accuracy for each empirical equation under large deflection. The errors were within $6 \%$ when compared to FEM results.

The constant rectangular cross section flexure hinge is widely used in the compact flexure-based mechanisms. Nevertheless, there are few methods to derive the compliance equations of the constant rectangular cross section flexure hinge. Furthermore, the stress concentration caused by changes in cross section can affect the distribution of the stress of flexure hinges. But the influences of the stress concentration have barely been taken into consideration when calculating the compliance of the flexure hinges [2023].

This paper addresses the constant rectangular cross section flexure hinge. Considering all geometrical parameters, the compliance equations with a relative wide range of $h / L$ and $t / L$ are derived. The influence of stress concentration caused by changes in cross section is taken into account during the whole analysis. Different ratios of $h / L$ and $t / L$ are investigated to overcome the influence induced by stress concentration. For verifying the validity of the empirical equations, FEM analyses and experiments of bridge-type microdisplacement amplification mechanisms have been done.

The remaining sections of this paper are organized as follows. Section 2 describes the compliance matrix. Section 3 analyzes the influence of stress concentration on compliance calculation errors including axial, shear, and bending compliance. In Section 4, empirical compliance equations are derived based on the exponential model in conjunction with consideration of all geometrical parameters of flexure hinges and the influence of the stress concentration on axial compliance calculation. In Section 5, empirical equations are used to analyze the input/output compliance and displacement amplification ratios of bridge-type microdisplacement amplification mechanisms. In Section 6, experiments have been done on experimental platform of bridge-type microdisplacement amplification mechanisms. Finally, conclusions are drawn in Section 7.

\section{The Compliance Matrix}

The constant rectangular cross section flexure hinge is a very common single-axis flexure hinge because it is simple to design and manufacture $[24,25]$. It can be made from a blank having rectangular rigid beams and it incorporates a constant rectangular cross section cutout [14]. Thus, it can be divided into two parts, the flexure hinge and the rigid beams, as shown in Figure 1.

The geometrical parameters of the constant rectangular cross section flexure hinge are shown in Figure 1 with its coordinate system whose origin is located at the free end of the hinge, and $x$-axis and $y$-axis are in the longitudinal and height directions, respectively. Geometrical dimensions include the hinge length $L$, the hinge thickness $t$, the side height $h$, the rigid beam width $W$, the total height $H$, and the total depth $D$.

For a single-axis flexure hinge, as illustrated in Figure 1, the loading at the end $a$ has six components: two bending moments, $M_{y}$ and $M_{z}$; two shearing forces, $F_{y}$ and $F_{z}$; one axial load, $F_{x}$; and one torsional moment, $M_{x}$. Considering the fact that constant rectangular cross section flexure hinges are used for planar compliant mechanisms, where all active and resistive loads are planar, only the in-plane components $M_{z}, F_{y}$, and $F_{x}$ have substantive effects on the flexure 

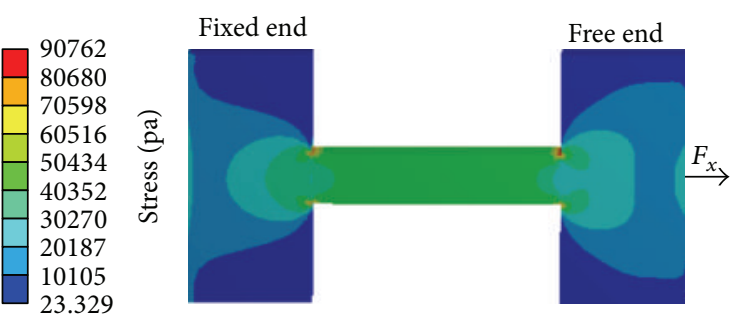

(a)

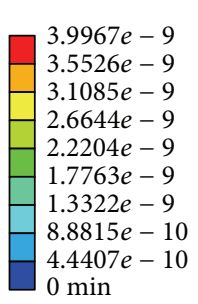

$0 \mathrm{~min}$

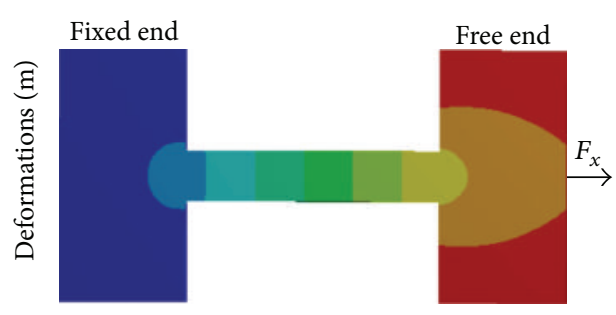

(b)

FIGURE 2: FEM results: (a) stress distribution caused by the unit axial force; (b) strain distribution caused by the unit axial force.

operation. Therefore, only the in-plane compliance is considered in this paper and the in-plane compliance matrix can be expressed as

$$
\mathbf{C}=\left[\begin{array}{ccc}
C_{x-F x} & 0 & 0 \\
0 & C_{y-F y} & C_{y-M z} \\
0 & C_{\alpha-F y} & C_{\alpha-M z}
\end{array}\right],
$$

where $\mathbf{C}_{m-n}$ is the compliance in the direction of $m$ caused by the force/moment $n$.

\section{Influence of the Stress Concentration}

3.1. Stress Concentration. In order to analyze the compliance calculation errors of the constant rectangular cross section flexure hinge, the stress and strain distribution caused by the unit axial force could be investigated, as shown in Figure 2. The geometrical properties of the constant rectangular cross section flexure hinge are given as $h=4 \mathrm{~mm}, L=10 \mathrm{~mm}$, $t=2 \mathrm{~mm}, W=5 \mathrm{~mm}$, and $D=10 \mathrm{~mm}$.

Basic theoretical calculation of the stress assumes that the components are smooth and have a uniform section and no irregularities. In practice virtually the constant rectangular cross section flexure hinge has changes in cross section along $x$-axis, which can change the stress distribution, so that the basic theoretical stress analysis equations no longer apply. Such changes in cross section cause a local increase of stress, referred to as stress concentration. We can see that the stress and strain of the surfaces with same colors are equal, and the surfaces with equal stress and strain are not vertical to $x$-axis in Figure 2, and they are also not parallel to each other, which is inconsistent with the basic theoretical stress assumptions.

Therefore, the stress and strain of the constant rectangular cross section flexure hinge are complicated and difficult to predict, which lead to the compliance calculation errors.

3.2. Influence on the Compliance Calculation. For studying the influence caused by the stress concentration on the compliance calculation of the constant rectangular cross section flexure hinge, the axial stress distribution obtained by FEM and basic theoretical stress assumptions along $x$-axis of a selected flexure hinge, which is under unite axial force, are compared in Figure 3. The geometrical properties of the selected flexure hinge are given as $h=4 \mathrm{~mm}, L=10 \mathrm{~mm}$, $t=2 \mathrm{~mm}, W=30 \mathrm{~mm}$, and $D=10 \mathrm{~mm}$.

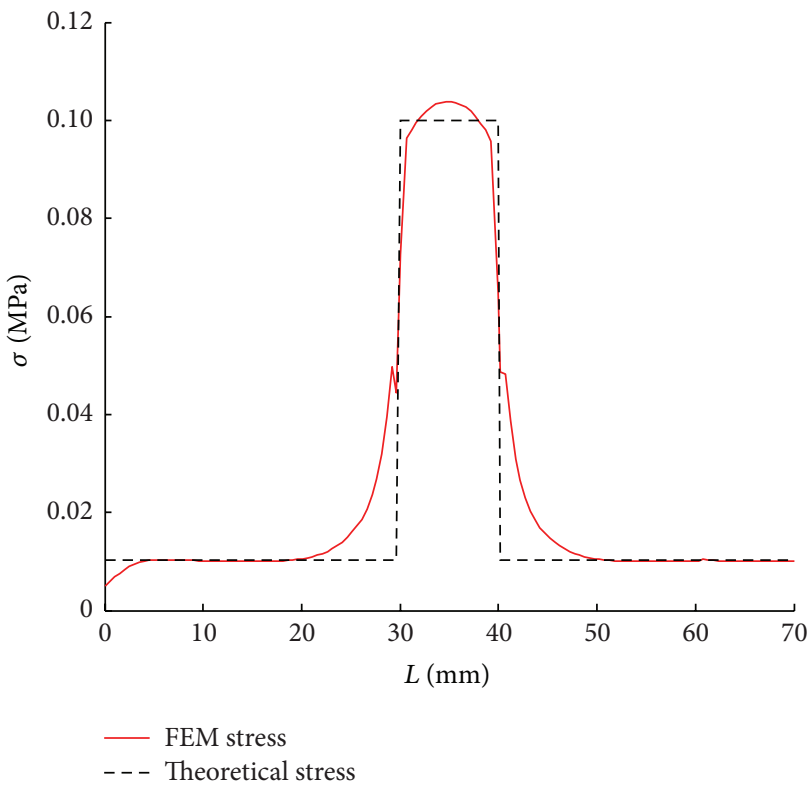

Figure 3: Axial stress distribution of the selected flexure hinge.

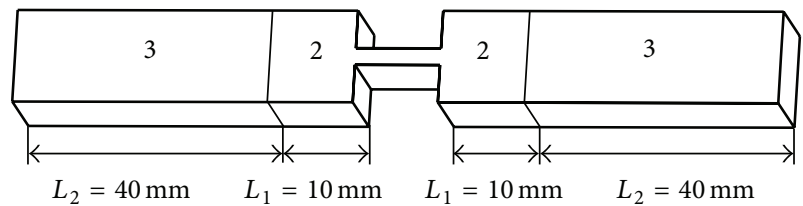

FIGURE 4: Flexure hinge with relative long rigid beams.

Considering the accuracy of the FEM, it is used as a benchmark for comparing with the theoretical stress. We can see that the benchmark stress is much larger than the theoretical stress when the cross section changes, which is in accordance with the stress concentration distribution caused by changes in the cross section.

The analysis above indicates that the stress concentration caused by changes in the cross section can affect the distribution of the stress and lead to compliance calculation errors. The constant rectangular cross section flexure hinge with relative long rigid beams is selected in Figure 4 to further investigate stress concentration. And it can be divided into three parts: the rectangle hinge, the rigid beams next to the hinge, and the rigid beams away from the hinge. 


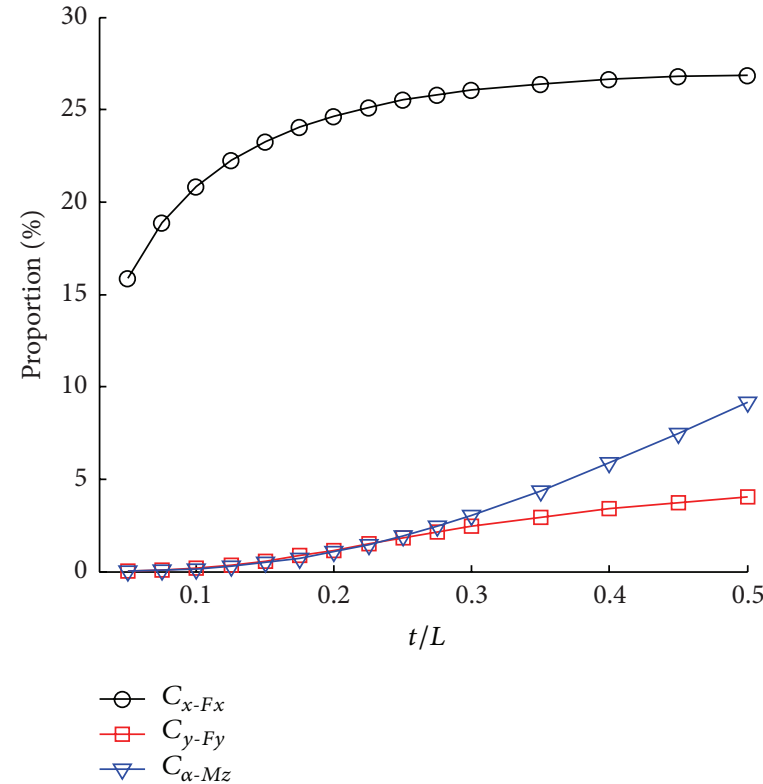

(a)

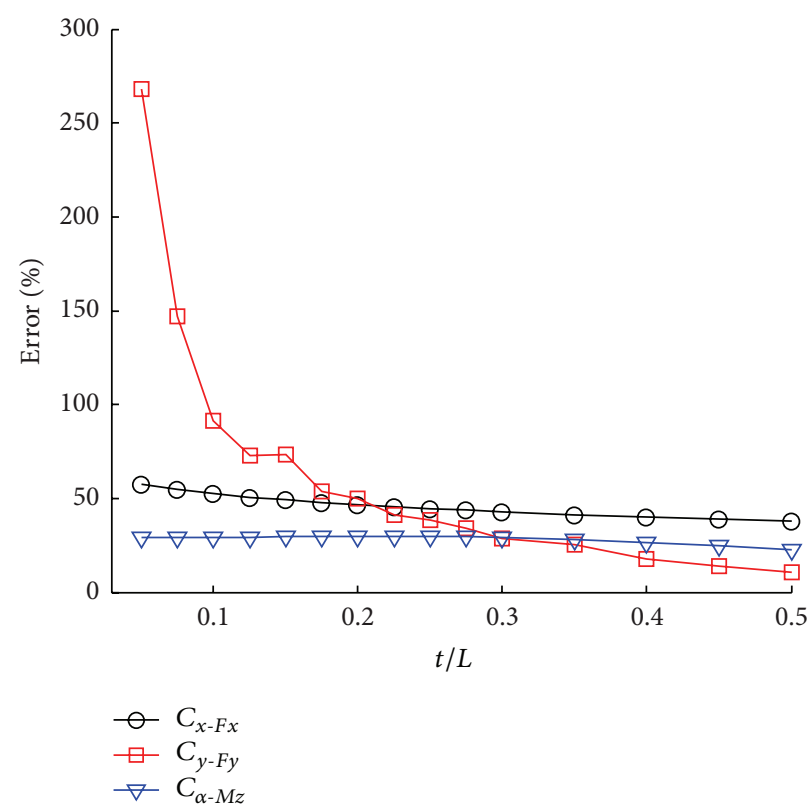

(b)

Figure 5: Compliance proportion and compliance calculation errors of the second part: (a) compliance proportion; (b) compliance calculation errors.

Then, the compliance proportion (the ratio of total compliance) and compliance calculation errors (the relative error between the theoretical value and the FEM value) of the second and third parts are discussed.

The compliance proportion and compliance calculation errors of the second part are shown in Figure 5.

One may see that, with increasing $t / L$, the compliance proportions of axial compliance $C_{x-F x}$, shear compliance $C_{y-F y}$, and bending compliance $C_{\alpha-M z}$ all increase, while the compliance calculation errors of them decrease. In addition, the compliance proportion of axial compliance is much larger than others', especially at large $t / L$ ratio. Moreover, the compliance calculation errors of them are relatively large, particularly the value of shear compliance, when $t / L$ is small $(0.05 \sim 0.2)$. The reason for this phenomenon is that the second parts next to the hinge are easily influenced by the stress concentration.

For investigating the influence of the stress concentration on the compliance calculation errors, the compliance proportion is multiplied by the compliance calculation errors as an evaluation factor. The evaluation factors of the second parts are shown in Figure 6. It can be seen that the evaluation factors of the axial compliance are much larger than those of shear compliance and bending compliance. In addition, with increasing $t / L$, the value of axis compliance at first increases; whereas $t / L$ increases continuously, the value decreases. Therefore, one can arrive at the conclusion that the stress concentration has great influence on the axial compliance calculation, while it has little influence on the shear compliance and bending compliance.

Similarly, the evaluation factors of the third parts are depicted in Figure 7. It is seen that the evaluation factors of each compliance component are small, especially the

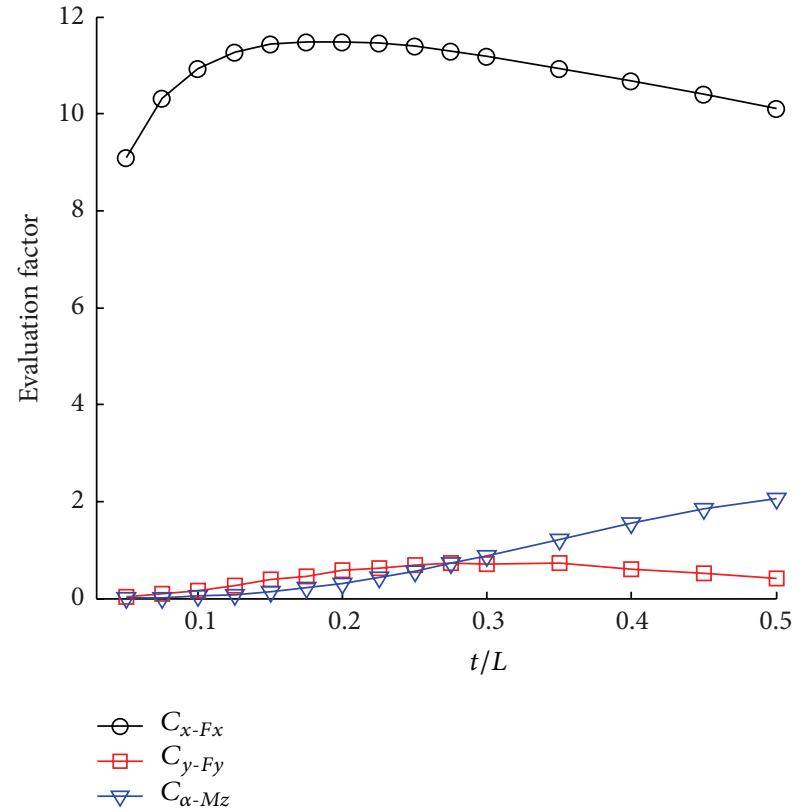

Figure 6: Trends of evaluation factors of the second part.

evaluation factor of the bending compliance. Therefore, the compliance calculation errors of the third part can be ignored. The reason for this phenomenon is that the third parts are away from the hinge and there are no changes in cross section.

The side height $h$ is another important geometrical parameter for the constant rectangular cross section flexure hinge. All the geometrical parameters of the constant rectangular cross section flexure hinge should be considered. Therefore, it is necessary to analyze the compliance proportion and 


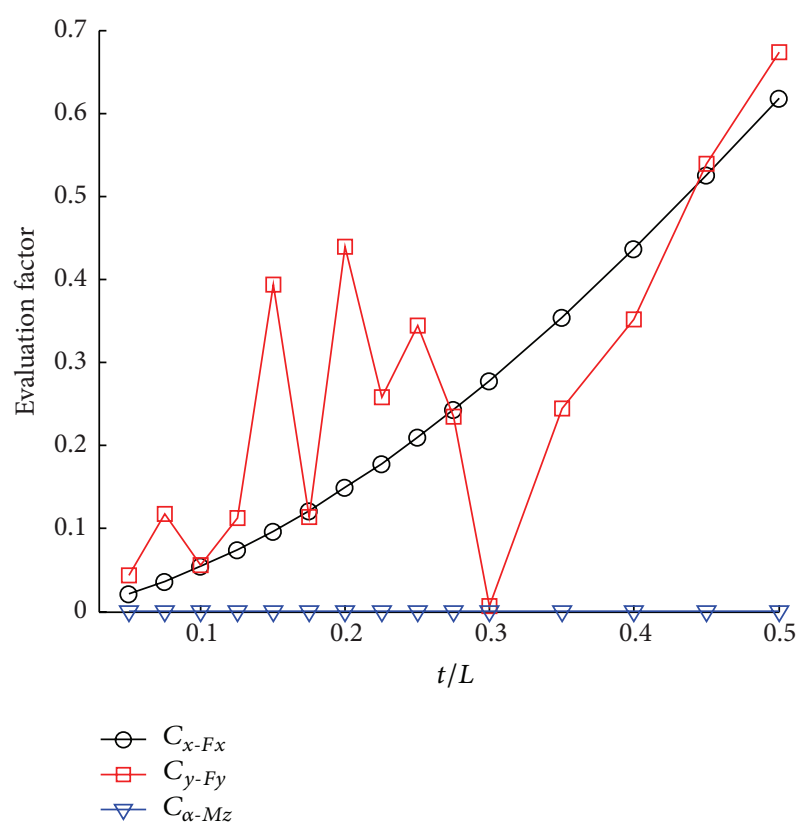

Figure 7: Trends of evaluation factors of the third part.

compliance calculation errors of the second and third parts when $h / L$ changed, and the results are shown in Figure 8.

It can be seen that the second part's evaluation factors of the axial compliance are much larger than those of shear compliance and bending compliance, especially at large $h / L$ ratio. In addition, the evaluation factors of shear compliance and bending compliance are relatively stable. By contract, the third part's evaluation factors of each compliance component are small, especially the evaluation factors of the shear compliance and bending compliance, while the evaluation factors of shear compliance are instable. It indicates that the stress concentration has great influence on the axial compliance calculation, while it has little influence on the shear compliance and bending compliance, when $h / L$ changed, which is the same as the conclusion obtained when $t / L$ changed.

\section{Empirical Compliance Equations}

Considering the influence caused by stress concentration on axial compliance calculation and all geometrical parameters of flexure hinges, empirical compliance equations of the constant rectangular cross section flexure hinge are derived in this part by means of FEM. The constant rectangular cross section has big stresses at its corners which can be much bigger than the other parts of the hinge. However, we are concerned with the deflections on $x$-axis (center line or axial line) as shown in Figure 1(b), and the deflections can be read by FEM directly. The analysis of stress concentration is to explain the phenomenon illustrated in Section 3.1 and arrive at the conclusion that the stress concentration has great influence on the axial compliance calculation of the whole constant rectangular cross section flexure hinge, while it has little influence on the shear compliance and bending compliance calculation. The processes of deriving empirical equations do not involve the stresses of the hinge. Therefore, the stresses at the corners cannot affect the accuracy of the empirical compliance equations.

4.1. Axial Compliance. The analyses above show that the stress concentration has a great influence on the axial compliance calculation of the whole constant rectangular cross section flexure hinge. Accordingly, the rigid beams next to the hinge should be considered when calculating the axial compliance equation. FEM is used to analyze the deflection of the constant rectangular cross section flexure hinge, and the restraint and load are shown in Figure 9. Only surface A is fixed, and the axial load is applied on surface D.

Figure 3 shows the axial stress distribution along $x$-axis of a constant rectangular cross section flexure hinge. It can be seen that the FEM curve can be divided into two regions: the constant region (below the straight line) over the whole hinge, which is almost in accordance with the basic theoretical stress curve, and the variable region (above the straight line), which follows the normal distribution curve. The area surrounded by the curve and the horizontal axis indicates the deflection, so that the total axial deflection can be expressed as

$$
\delta=\delta_{a}+\delta_{b}
$$

where $\delta$ is the total axial deflection, $\delta_{a}$ is the deflection of the constant region, and $\delta_{b}$ is the deflection of the variable region.

The uniform distributed load is applied on surface D of the constant rectangular cross section flexure hinge to keep the deflection of the rigid beams constant, and the deflection of the constant region can be expressed as

$$
\delta_{a}=\frac{F L_{a}}{E A}=\frac{F(L+2 W)}{E D(2 h+t)}=\frac{P L_{a}}{E},
$$

where $F$ is the axial load, $A$ is the cross-sectional area, $L_{a}$ is the total length, and $E$ is Young's modulus.

According to the theories of mechanisms of materials, the equation which involved all the geometrical parameters affecting the deflection of the variable region can be expressed as

$$
\delta_{b}=K \frac{P L^{m} h^{u}}{E t^{n}}
$$

where $K$ is the constant coefficient which is independent of the geometrical parameters, material properties, and the loads; $m, n$, and $u$ are the indexes corresponding to the geometrical parameters.

We are concerned with the deflections on $x$-axis, and the deflections can be read by FEM directly. The geometrical parameters are taken as $W=40 \mathrm{~mm}$ and $D=10 \mathrm{~mm}$. Firstly, the hinge length is $10 \mathrm{~mm}$, the side height is $4.5 \mathrm{~mm}$, and the value of $t$ is varied from $0.5 \mathrm{~mm}$ to $5 \mathrm{~mm}$ to analyze the relationship between $t$ and $\delta_{b}$, as shown in Figure 10. We can see that the curve indicates the exponential relationship between them. The logarithm of the values of the horizontal and vertical coordinates is obtained, and then the least square method is applied for getting the value of the indexes. In order to obtain the uniform dimension, the index is revised, 


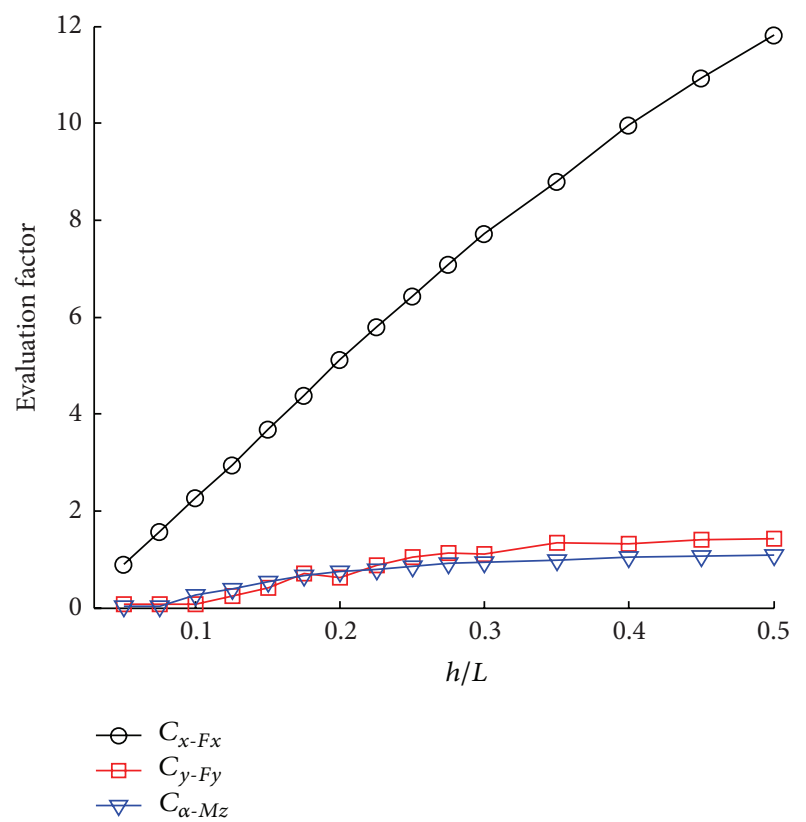

(a)

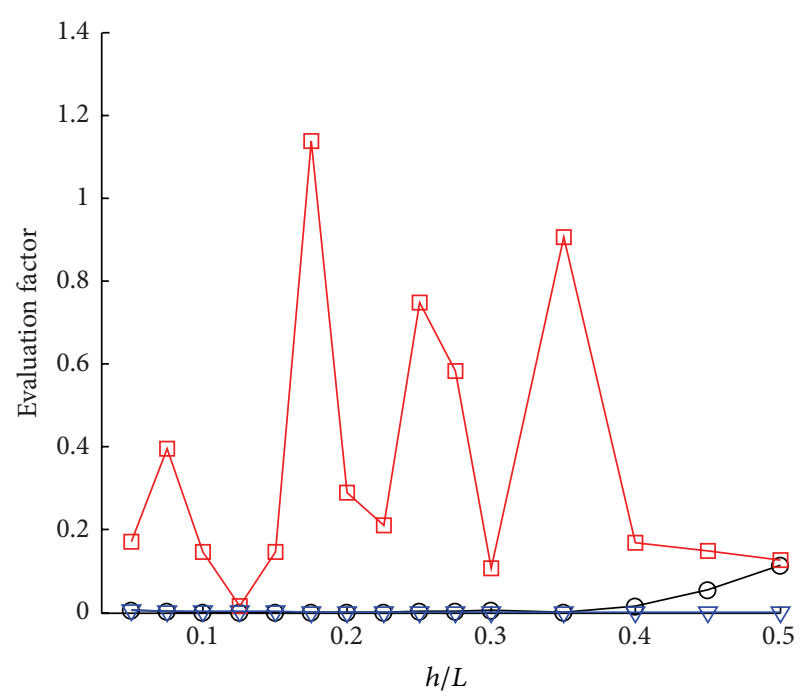

$-C_{x-F x}$
$\square C_{y-F y}$
$\nabla C_{\alpha-M z}$

FIGURE 8: Trends of evaluation factors: (a) the second part; (b) the third part.

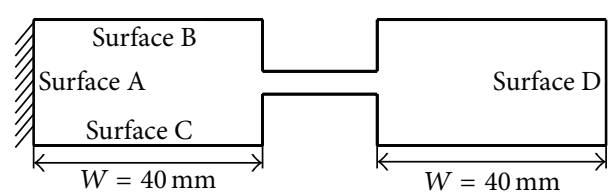

FIGURE 9: Restraint and load of the constant rectangular cross section flexure hinge.

and $n=0.8224$. Then, the hinge length is $10 \mathrm{~mm}$, the hinge thickness is $1 \mathrm{~mm}$, and the value of $h$ is varied from $0.5 \mathrm{~mm}$ to $5 \mathrm{~mm}$ to analyze the relationship between $h$ and $\delta_{b}$. Finally, the side height is $4.5 \mathrm{~mm}$, the hinge thickness is $1 \mathrm{~mm}$, and the value of $L$ is varied from $2 \mathrm{~mm}$ to $20 \mathrm{~mm}$ to analyze the relationship between $L$ and $\delta_{b}$. Similarly, the indexes $u$ and $m$ can be obtained as 1.0709 and 0.7515 , respectively.

According to the principle that the corresponding mean square deviation of $\delta_{b}$ is minimum, the constant coefficient can be obtained as $K_{t}=3.9880, K_{L}=4.0905$, and $K_{h}=$ 3.9356 in terms of the relationships $\delta_{b}-t, \delta_{b}-L$, and $\delta_{b}-h$, and then the average value can be calculated as $K=4$.

Hence, the total axial deflection and the axial compliance of the constant rectangular cross section flexure hinge are given as follows:

$$
\begin{gathered}
\delta=\delta_{a}+\delta_{b}=\frac{F}{E D(2 h+t)}\left[4 \cdot h \cdot\left(\frac{h}{t}\right)^{0.0709}\right. \\
\left.\cdot\left(\frac{L}{t}\right)^{0.7515}+L+2 W\right],
\end{gathered}
$$

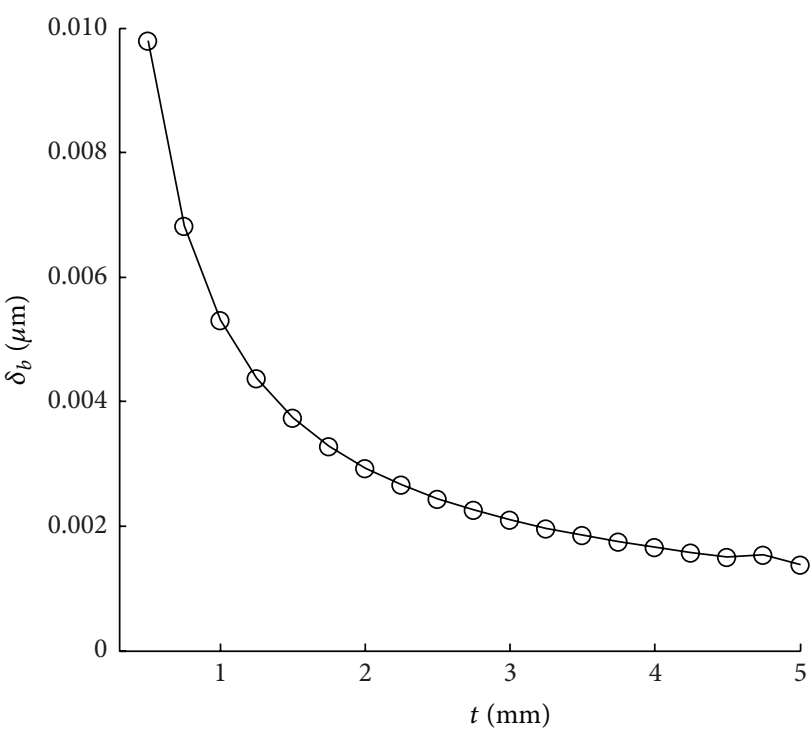

FIgURE 10: Relationship between $\delta_{b}$ and $t$.

$$
\begin{aligned}
& C_{x-F x}=\frac{1}{E D(2 h+t)}\left[4 \cdot h \cdot\left(\frac{h}{t}\right)^{0.0709} \cdot\left(\frac{L}{t}\right)^{0.7515}+L\right. \\
& +2 W] .
\end{aligned}
$$

4.2. Bending Compliance. The analyses above show that the stress concentration has little influence on the bending compliance and shear compliance calculation. Accordingly, the 


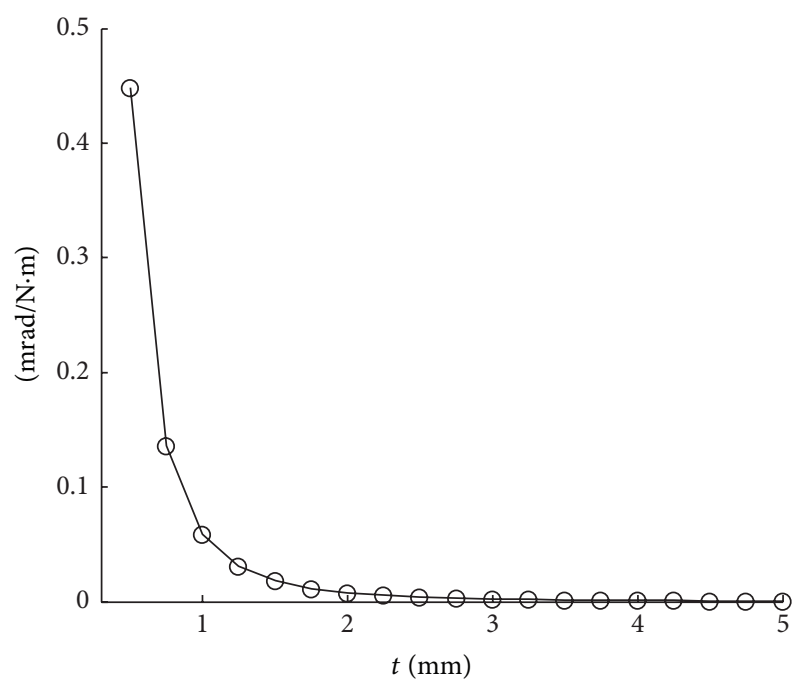

FIGURE 11: Relationship between $\theta$ and $t$.

stress concentration can be ignored, and only flexure hinge is chosen to calculate the bending compliance. Therefore, the restraint and load are shown in Figure 9, surface A, surface B, and surface $C$ are all fixed, and the bending moment is applied on surface D.

According to the theories of mechanisms of materials, the equation which involved all the geometrical parameters affecting $\theta$ can be expressed as

$$
\theta=K \cdot \frac{M}{E D} \cdot \frac{L^{m}}{t^{n}}=K \cdot \frac{P}{E} \cdot \frac{L^{m}}{t^{n}}
$$

where $K$ is the constant coefficient which is independent of the geometrical parameters, material properties, and the loads; $m$ and $n$ are the indexes corresponding to the geometrical parameters.

The deflections along $x$-axis can be read by FEM directly. The geometrical parameters are taken as $W=40 \mathrm{~mm}$ and $D=10 \mathrm{~mm}$. Firstly, the hinge length is $10 \mathrm{~mm}$, the side height is $4.5 \mathrm{~mm}$, and the value of $t$ is varied from $0.5 \mathrm{~mm}$ to $5 \mathrm{~mm}$ to analyze the relationship between $t$ and $\theta$, as shown in Figure 11. Then, the relationship between $L$ and $\theta$ can also be analyzed with the same geometrical parameters as the ones of axial compliance calculation. Similarly, the indexes $m$ and $n$ can be obtained as 0.8856 and 2.8856 , respectively.

Similarly, according to the relationships $\theta$ - $t$ and $\theta-L$ illustrated in Figure 11, the constant coefficient can be obtained as $K_{t}=16.5202$ and $K_{L}=16.5498$, and the average value can be calculated as $K=16.5350$. Hence, the bending compliance of the constant rectangular cross section flexure hinge can be expressed as

$$
C_{\alpha-M z}=\frac{16.5350}{E D t^{2}} \cdot\left(\frac{L}{t}\right)^{0.8856} .
$$

Similarly, the compliance equation for $C_{y-M z}$ can be written as

$$
C_{y-M z}=\frac{8.3493}{E D t} \cdot\left(\frac{L}{t}\right)^{1.8842}
$$

4.3. Shear Compliance. The analyses above show that the stress concentration has little influence on the shear compliance calculation. Similarly, only flexure hinge is chosen to calculate the shear compliance. Similarly, the compliance equations of $C_{y-F y}$ and $C_{\alpha-F y}$ can be expressed as

$$
\begin{aligned}
C_{y-F y}= & \frac{5.2749}{E D} \cdot\left(\frac{h}{t}\right)^{0.0124} \cdot\left(\frac{L}{t}\right)^{2.8996}+\frac{\alpha \cdot E}{G} \\
& \cdot C_{x-F x}, \\
C_{\alpha-F y}= & \frac{8.3802}{E D t} \cdot\left(\frac{L}{t}\right)^{1.8826} .
\end{aligned}
$$

4.4. Validation. In order to verify the accuracy and validity of the empirical compliance equations derived above, the errors between the results calculated by empirical equations and FEM are analyzed, as shown in Figure 12(a). The geometrical properties of the selected flexure hinge are given as $h=4 \mathrm{~mm}$, $L=20 \mathrm{~mm}, W=30 \mathrm{~mm}$, and $D=10 \mathrm{~mm}$, and the value of $t$ is varied from $0.5 \mathrm{~mm}$ to $5 \mathrm{~mm}$. It can be seen that the errors are below $10 \%$, which proves that the value calculated by empirical equations is almost coincident with the FEM value. In addition, the errors of $C_{\alpha-F y}$ and $C_{y-M z}$ are almost the same, which is in accordance with the compliance equation, $C_{\alpha-F y}=C_{y-M z}$.

As shown in Figure 12(b), the compliance calculation errors of present compliance equations depicted in literature [1] are compared, and the average errors are above $10 \%$. The comparisons between Figure 12(a) and Figure 12(b) show that the empirical compliance equations are more accurate than present closed-form compliance equations for a wide range of $t / L$ (from 0.1 to 0.5 ).

\section{Applications}

In order to confirm the validity of the empirical equations mentioned above, it is necessary to carry on the compliance and displacement amplification ratio analysis to the bridgetype microdisplacement amplification mechanism. As we all know, the microdisplacement amplification mechanism processed as a whole on a piece of metal with wire cutting technology recently has a compact structure and a large displacement amplification ratio [26-29].

The conclusion above shows that the stress concentration has great influence on the axial compliance of the constant rectangular cross section flexure hinge. For improving the calculation accuracy, rectangle hinge with rigid beams is regarded as a whole part.

The structure/geometrical parameters/restraint and load of the bridge-type microdisplacement amplification mechanism are shown in Figure 13. The geometrical parameters of the amplification mechanism are given as $a=37.5 \mathrm{~mm}$, $b=25 \mathrm{~mm}, d=30 \mathrm{~mm}$, and $l=135 \mathrm{~mm}$, and the geometrical parameters of the constant rectangular cross section flexure hinge are given as $h=4.5 \mathrm{~mm}, L=10 \mathrm{~mm}, t=2 \mathrm{~mm}$, $W=5 \mathrm{~mm}$, and $D=10 \mathrm{~mm}$. The results calculated by FEM can be regarded as relatively accurate, and FEM is used to analyze the static mechanics of the bridge-type mechanism. 


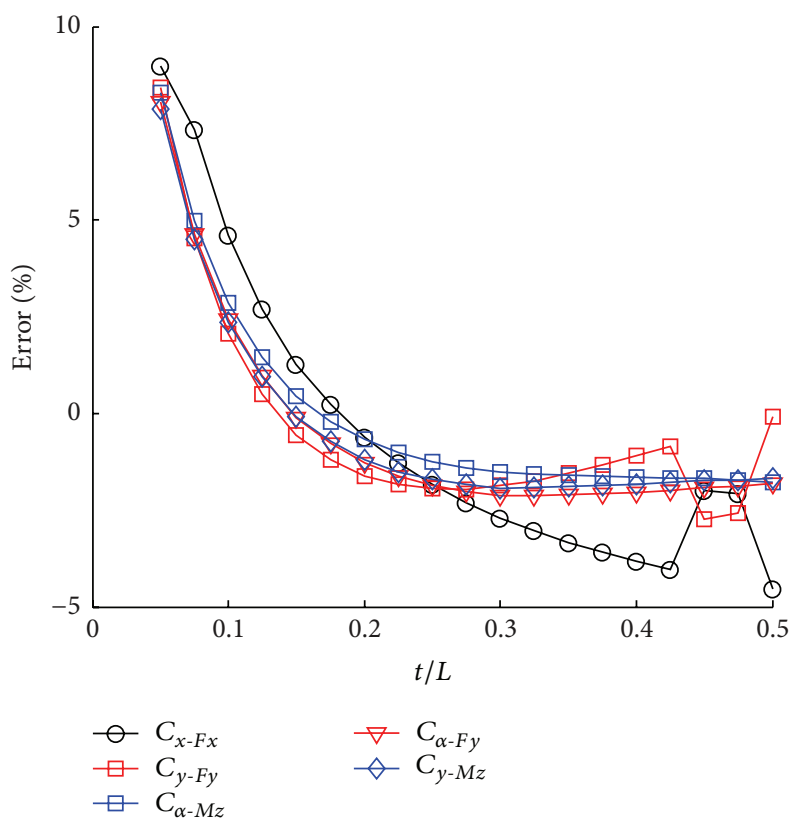

(a)

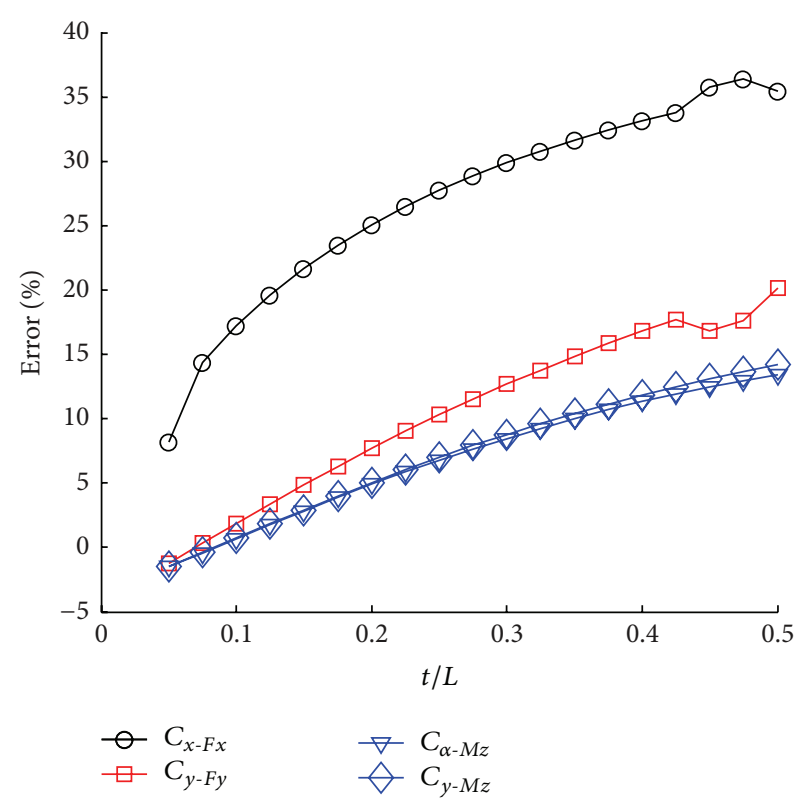

(b)

FIGURE 12: Compliance calculation errors: (a) empirical compliance equations; (b) present compliance equations.

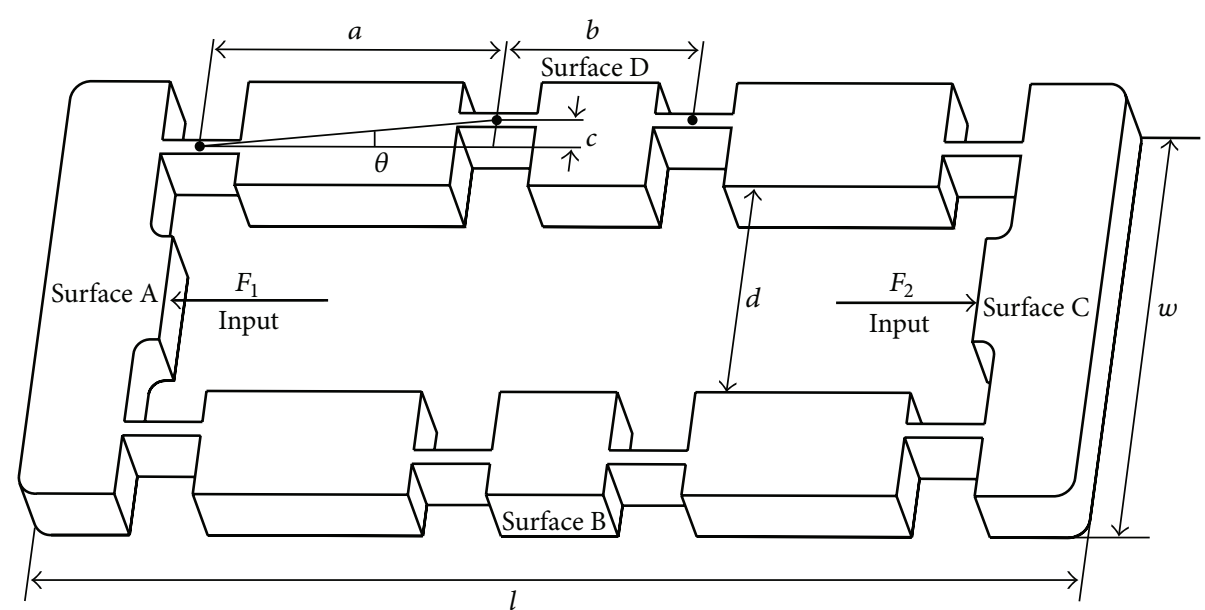

FIGURE 13: Structure/geometrical parameters/restraint and load of amplify mechanism.

Mesh density could affect the model simulation accuracy and the model simulation time step. For similar mesh quality, the model with high mesh density has more elements and smaller simulation time steps and thus increases calculation accuracy and computational costs, respectively. In this work, FEM is performed using ANSYS Workbench software. Hexahedral meshes and appropriate mesh density are selected. Surface D is fixed. There is a displacement output on surface $C$, when imposing a force on surfaces A and B.

The input/output compliance and displacement amplification ratio, which mainly depend on angle $\theta$, are the important performances for this mechanism. The relationship between the input/output compliance of this mechanism and angle $\theta$ is firstly investigated. Figure 14 shows the results calculated by the analytical model and FEM. We can see that the input compliance and output compliance both increase with increasing angle $\theta$, and the output compliance and angle $\theta$ maintain a linear relationship. Furthermore, the theoretical value is almost similar to the FEM value.

Figure 15 shows the relationship between the displacement amplification ratio and angle $\theta$ calculated by analytical model and FEM. It can be seen that, with increasing angle $\theta$, the displacement amplification ratio at first increases sharply, and it has a maximum value and $\theta$ reaches a threshold value. Whereas $\theta$ increases continuously, the displacement amplification ratio decreases. Through the comparison between the displacement amplification calculated by analytical model and FEM, we can see that the FEM value is obviously smaller 


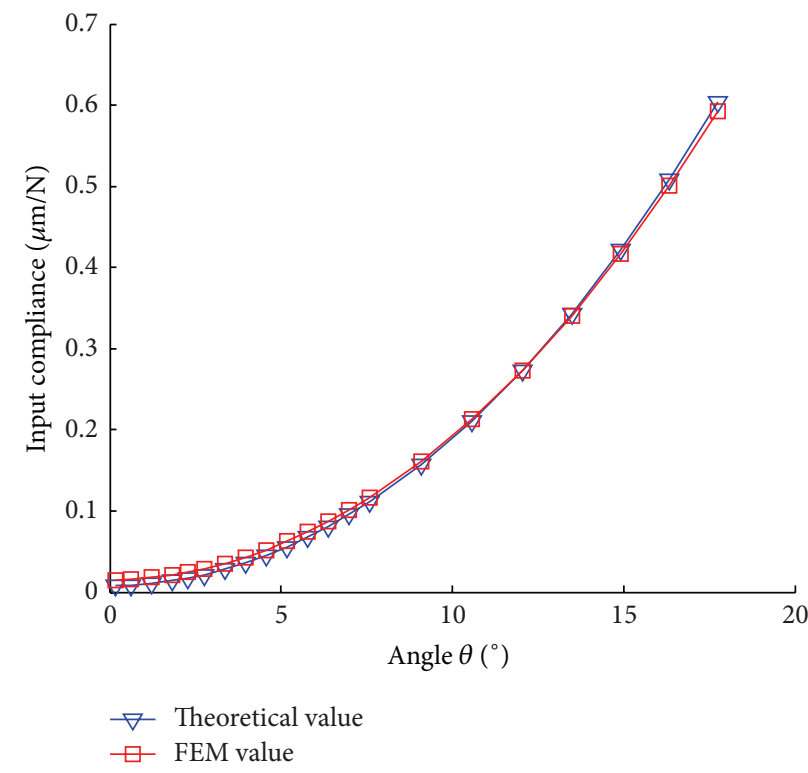

(a)

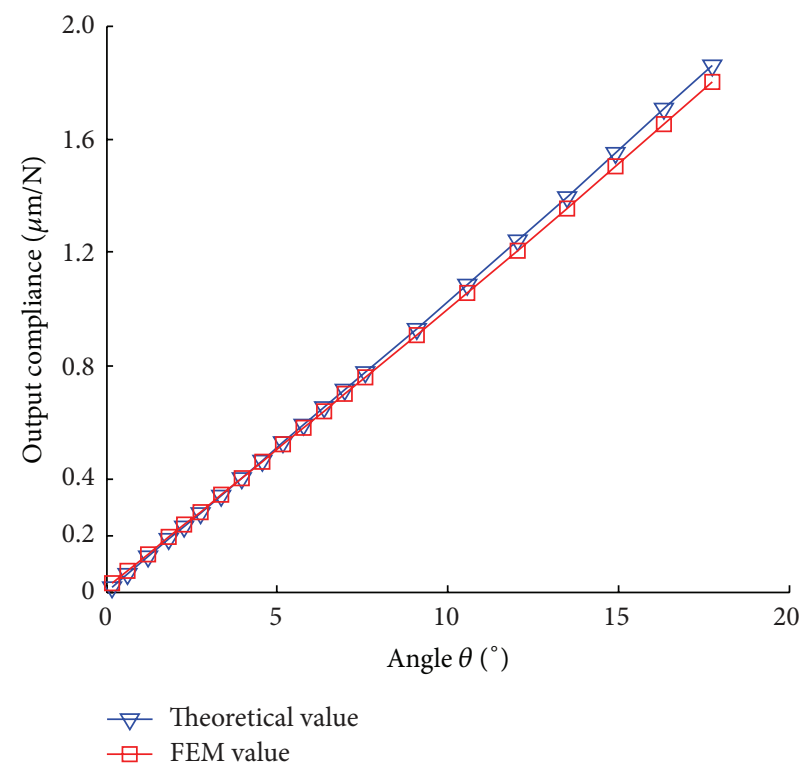

(b)

FIGURE 14: Compliance of the amplification mechanism: (a) input compliance; (b) output compliance.

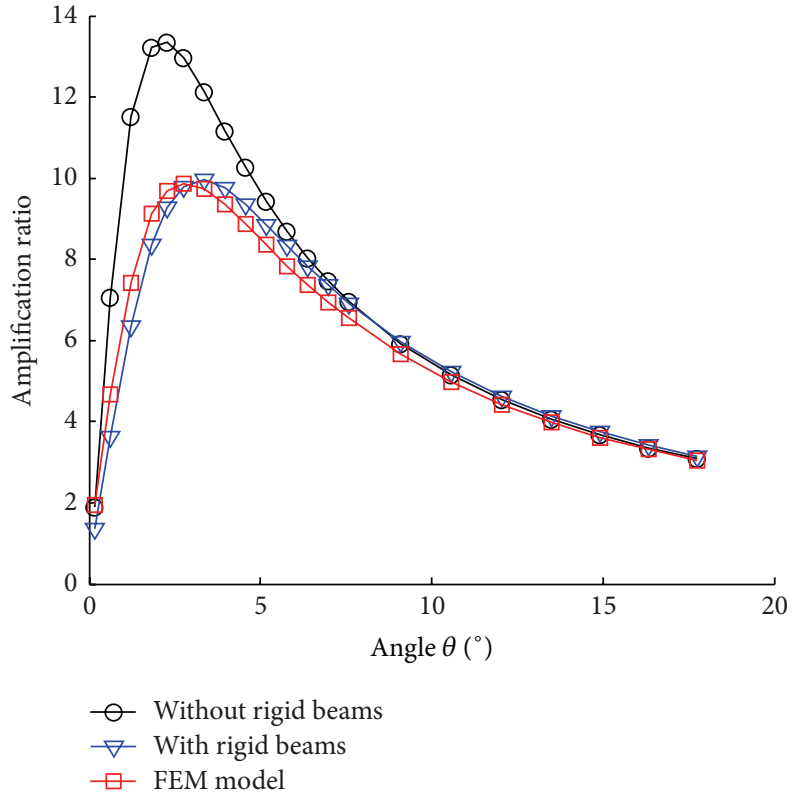

Figure 15: Displacement amplification ratio.

than the theoretical value when angle $\theta$ is small. In fact, this is mainly because the rigid beams are not absolute rigid components which also undergo elastic deflection. When the angle $\theta$ is small enough, the deflection of the rigid beams cannot be neglected.

Then, the deflection of the rigid beams is taken into account, and the displacement amplification ratio is calculated and shown in Figure 15. We can see that the theoretical value is almost similar to the FEM value, which proves the accuracy of the empirical equations.

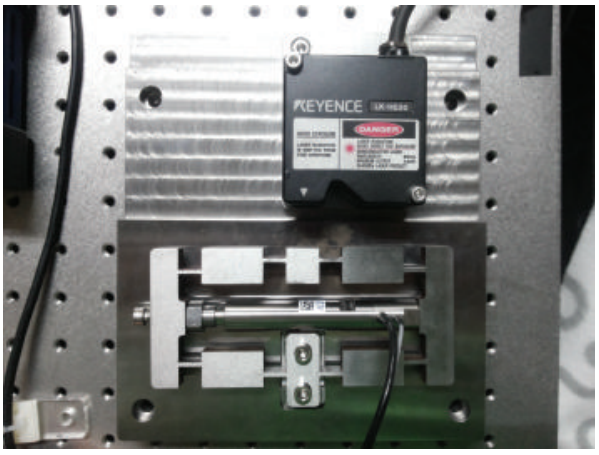

FIGURE 16: Bridge-type mechanism and experimental setup.

\section{Experiments}

To verify the accuracy of the empirical equations, an experimental platform of the bridge-type microdisplacement amplification mechanism is designed and constructed to test the displacement amplification ratio.

Two sample pieces of bridge-type microdisplacement amplification mechanism shown as Figure 16 were processed by NC low-speed WEDM. The geometrical parameters of each sample are shown above: only the angle $\theta$ is different: one is 3.05 and the other is 7.60. The material of the samples is $45 \#$ steel, the elastic modulus $E$ is $210 \mathrm{GPa}$, and their Poisson's Ratio, $\mu$, is 0.269 .

P-842.40 preloaded piezoelectric actuator produced by Power Integrations Co., Ltd. is selected as microdisplacement driver, with a voltage range of $0-100 \mathrm{~V}$, nominal displacement of $60 \mu \mathrm{m}$, and closed-loop resolution of $0.6 \mathrm{~nm}$. The PZT is driven by a microactuator drive power (E-621 LVPZT controller from Power Integrations Co., Ltd.), with 
TABLE 1: Displacement amplification ratios.

\begin{tabular}{lcccc}
\hline & $\theta$ & FEM value & $\begin{array}{c}\text { Theoretical } \\
\text { value }\end{array}$ & $\begin{array}{c}\text { Experimental } \\
\text { value }\end{array}$ \\
\hline Sample 1 & 3.05 & 9.93 & 10.35 & 9.37 \\
Sample 2 & 7.60 & 6.54 & 7 & 6.21 \\
\hline
\end{tabular}

an external input voltage of $-2 \sim 12 \mathrm{~V}$ and output voltage of $-20 \sim 120$ V. Furthermore, one LK-H025 laser displacement sensor produced by KEYENCE Co., Ltd. is used as detecting device, which has a measuring range of $\pm 3 \mathrm{~mm}$, and its repeatability precision is $0.020 \mu \mathrm{m}$. The piezoelectric actuator is preloaded through the screw mounted at the mechanism. The preloading force is used to eliminate the influence of the clearance, and it is difficult to be measured. The experimental result could increase with increasing preloading force when there is clearance. By contrast, it could be almost constant when there is no clearance. Of course, in order to generate displacements from the actuator, the preloading force could not be too large. Figure 16 is the photograph of the mechanism and experimental setups, and the amplification ratios are shown in Table 1.

It is obvious that the theoretical value is similar to FEM value and experimental value, which proves the accuracy of the empirical equations.

\section{Conclusions}

In this paper, empirical compliance equations with a relative wide range of $h / L$ and $t / L$ are derived based on the exponential model. The input/output compliance and displacement amplification ratios of the bridge-type microdisplacement amplification mechanism are analyzed by analytical model and FEM. An experiment on displacement amplification ratios has been done to verify the accuracy of the empirical compliance equations. Therefore, we can draw the following conclusions:

(1) The stress concentration has great influence on the axial compliance calculation of the whole constant rectangular cross section flexure hinge, while it has little influence on the shear compliance and bending compliance calculation.

(2) Besides the hinge length $L$ and hinge thickness $t$, the side height $h$ should be considered when calculating the compliance of the constant rectangular cross section flexure hinge, which is included in the axial and shear compliance empirical equations.

(3) Empirical compliance equations can be derived by exponential model, and these equations are verified to be valid and accurate compared with FEM and present compliance equations through a relative wide range of $h / L$ and $t / L$.

(4) Theoretical displacement amplification calculated by empirical compliance equations is similar to FEM values and experimental values, which proves the accuracy of these equations.

\section{Competing Interests}

The authors declare that they have no competing interests.

\section{Acknowledgments}

This work was supported by the National Basic Research Program of China (Grant no. 2011CB302400), the National Science Foundation of China (Grant no. 51275260), and the National Science and Technology Major Project of China (Grant no. 2015ZX04001002 and Grant no. 2015ZX04014021).

\section{References}

[1] N. Lobontiu, Compliant Mechanisms: Design of Flexure Hinges, CRC Press, New York, NY, USA, 2010.

[2] Y. Tian, B. Shirinzadeh, D. Zhang, and Y. Zhong, "Three flexure hinges for compliant mechanism designs based on dimensionless graph analysis," Precision Engineering, vol. 34, no. 1, pp. 92$100,2010$.

[3] M. N. M. Zubir, B. Shirinzadeh, and Y. Tian, "Development of a novel flexure-based microgripper for high precision microobject manipulation," Sensors and Actuators A: Physical, vol. 150, no. 2, pp. 257-266, 2009.

[4] Y. $\mathrm{Li}$ and Q. Xu, "A novel design and analysis of a 2-DOF compliant parallel micromanipulator for nanomanipulation," IEEE Transactions on Automation Science and Engineering, vol. 3, no. 3, pp. 247-254, 2006.

[5] Y. Li and Q. Xu, "Design of a new decoupled XY flexure parallel kinematic manipulator with actuator isolation," in Proceedings of the IEEE/RSJ International Conference on Intelligent Robots and Systems (IROS '08), pp. 470-475, IEEE, Nice, France, September 2008.

[6] Q. Xu, "Design and development of a flexure-based dual-stage nanopositioning system with minimum interference behavior," IEEE Transactions on Automation Science and Engineering, vol. 9, no. 3, pp. 554-563, 2012.

[7] L.-J. Lai, G.-Y. Gu, and L.-M. Zhu, "Design and control of a decoupled two degree of freedom translational parallel micropositioning stage," Review of Scientific Instruments, vol. 83, no. 4, Article ID 045105, 2012.

[8] Y. Li, S. Xiao, L. Xi, and Z. Wu, "Design, modeling, control and experiment for a 2-DOF compliant micro-motion stage," International Journal of Precision Engineering and Manufacturing, vol. 15, no. 4, pp. 735-744, 2014.

[9] P. R. Ouyang, R. C. Tjiptoprodjo, W. J. Zhang, and G. S. Yang, "Micro-motion devices technology: the state of arts review," International Journal of Advanced Manufacturing Technology, vol. 38, no. 5-6, pp. 463-478, 2008.

[10] Y. K. Yong and T.-F. Lu, "The effect of the accuracies of flexure hinge equations on the output compliances of planar micromotion stages," Mechanism and Machine Theory, vol. 43, no. 3, pp. 347-363, 2008.

[11] Y. K. Yong and T.-F. Lu, "Comparison of circular flexure hinge design equations and the derivation of empirical stiffness formulations," in Proceedings of the IEEE/ASME International Conference on Advanced Intelligent Mechatronics (AIM '09), pp. 510-515, Singapore, July 2009.

[12] J. M. Paros, "How to design flexure hinges," Machine Design, vol. 37, pp. 151-156, 1965. 
[13] Y. Wu and Z. Zhou, "Design calculations for flexure hinges," Review of Scientific Instruments, vol. 73, no. 8, pp. 3101-3106, 2002.

[14] W. O. Schotborgh, F. G. M. Kokkeler, H. Tragter, and F. J. A. M. Van Houten, "Dimensionless design graphs for flexure elements and a comparison between three flexure elements," Precision Engineering, vol. 29, no. 1, pp. 41-47, 2005.

[15] N. Lobontiu, E. Garcia, M. Hardau, and N. Bal, "Stiffness characterization of corner-filleted flexure hinges," Review of Scientific Instruments, vol. 75, no. 11, pp. 4896-4905, 2004.

[16] N. Lobontiu, J. S. N. Paine, E. Garcia, and M. Goldfarb, "Cornerfilleted flexure hinges," Journal of Mechanical Design, vol. 123, no. 3, pp. 346-352, 2001.

[17] Y. M. Tseytlin, "Notch flexure hinges: an effective theory," Review of Scientific Instruments, vol. 73, no. 9, pp. 3363-3368, 2002.

[18] M. Acer and A. Sabanovic, "Comparison of circular flexure hinge compliance modeling methods," in Proceedings of the IEEE International Conference on Mechatronics (ICM '11), pp. 271-276, IEEE, Istanbul, Turkey, April 2011.

[19] Q. Meng, Y. Li, and J. Xu, "New empirical stiffness equations for corner-filleted flexure hinges," Mechanical Sciences, vol. 4, no. 2, pp. 345-356, 2013.

[20] S. T. Smith, V. G. Badami, J. S. Dale, and Y. Xu, "Elliptical flexure hinges," Review of Scientific Instruments, vol. 68, no. 3, pp. 14741483, 1997.

[21] N. Lobontiu, J. S. N. Paine, E. Garcia, and M. Goldfarb, "Design of symmetric conic-section flexure hinges based on closedform compliance equations," Mechanism and Machine Theory, vol. 37, no. 5, pp. 477-498, 2002.

[22] G.-M. Chen, J.-Y. Jia, and Z.-W. Li, "Right-circular cornerfilleted flexure hinges," in Proceedings of the IEEE Conference on Automation Science and Engineering, pp. 249-253, IEEE, Edmonton, Canada, August 2005.

[23] S. Linß, T. Erbe, and L. Zentner, "On polynomial flexure hinges for increased deflection and an approach for simplified manufacturing," in Proceedings of the 13th World Congress in Mechanisms and Machine Science, pp. 1-9, Guanajuato, Mexico, June 2011.

[24] Q. Xu, "New flexure parallel-kinematic micropositioning system with large workspace," IEEE Transactions on Robotics, vol. 28, no. 2, pp. 478-491, 2012.

[25] Q. Xu, "Design and development of a compact flexure-based $\mathrm{XY}$ precision positioning system with centimeter range," IEEE Transactions on Industrial Electronics, vol. 61, no. 2, pp. 893-903, 2014.

[26] N. Lobontiu and E. Garcia, "Analytical model of displacement amplification and stiffness optimization for a class of flexurebased compliant mechanisms," Computers and Structures, vol. 81, no. 32, pp. 2797-2810, 2003.

[27] H.-W. Ma, S.-M. Yao, L.-Q. Wang, and Z. Zhong, "Analysis of the displacement amplification ratio of bridge-type flexure hinge," Sensors and Actuators A: Physical, vol. 132, no. 2, pp. 730-736, 2006.

[28] Q. Xu and Y. Li, "Analytical modeling, optimization and testing of a compound bridge-type compliant displacement amplifier," Mechanism and Machine Theory, vol. 46, no. 2, pp. 183-200, 2011.

[29] G. Ye, W. Li, Y.-Q. Wang, X.-F. Yang, and L. Yu, "Kinematics analysis of bridge-type micro-displacement mechanism based on flexure hinge," in Proceedings of the IEEE International
Conference on Information and Automation (ICIA '10), pp. 6670, IEEE, Harbin, China, June 2010. 


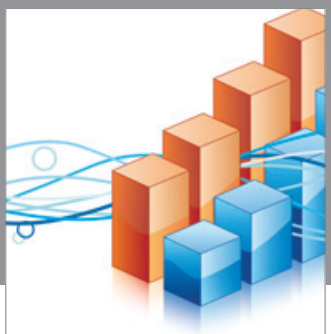

Advances in

Operations Research

vatem alat4

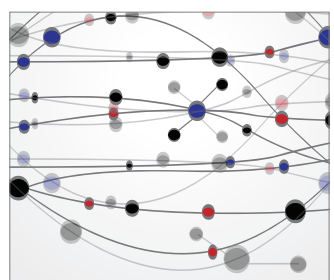

\section{The Scientific} World Journal
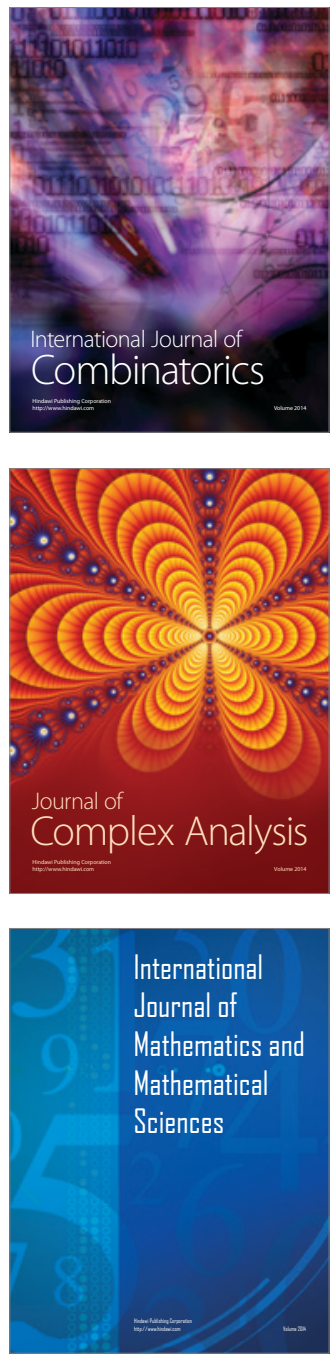
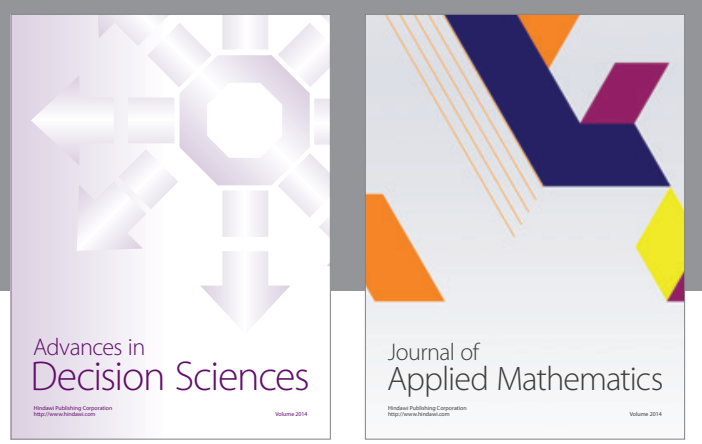

Algebra

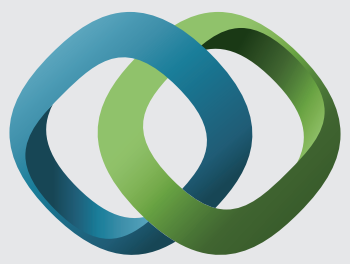

\section{Hindawi}

Submit your manuscripts at

http://www.hindawi.com
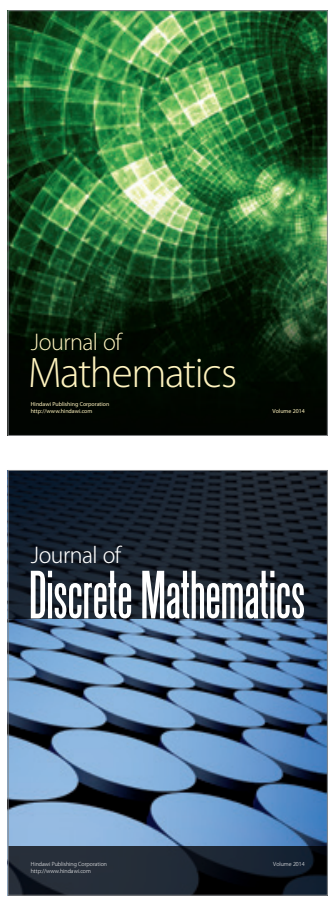

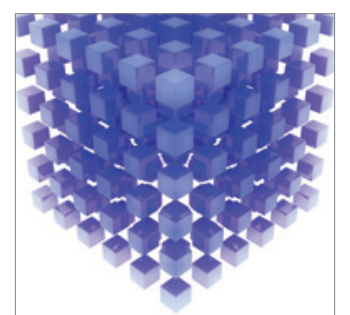

Mathematical Problems in Engineering
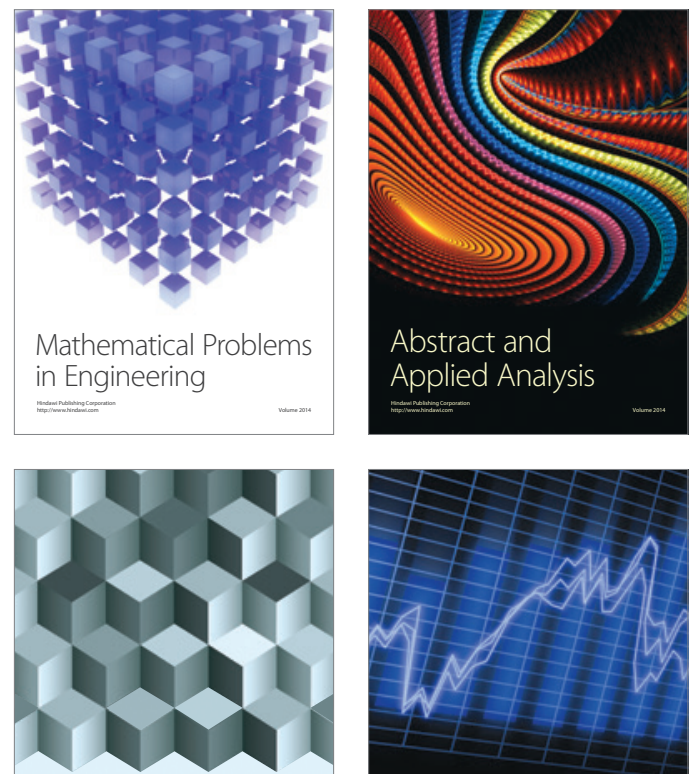

Journal of

Function Spaces

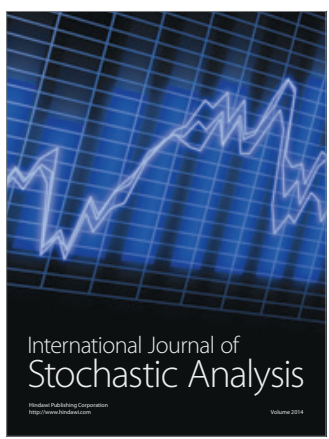

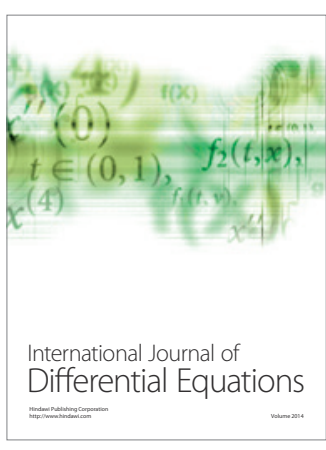
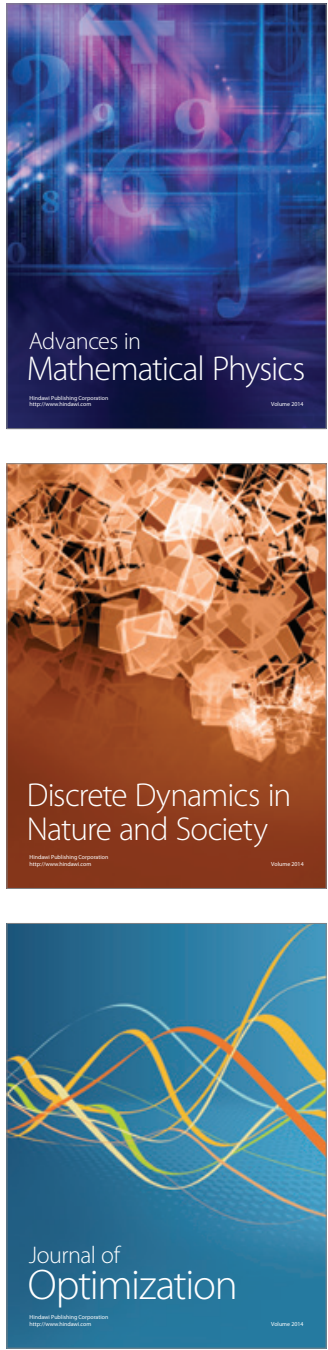